\title{
Quantum oscillations in a stack of superconducting cylinders in a magnetic field: crossover from the Aharonov-Bohm to the Little-Parks regime
}

\author{
V. M. Gvozdikov \\ Department of Physics, Kharkov State University, 61077, Svobody Sq. 4, Kharkov, Ukraine \\ E-mail: Vladimir.M.Gvozdikov@univer.kharkov.ua \\ Received February 1, 2000, revised March 15, 2000
}

\begin{abstract}
The Aharonov-Bohm (AB) oscillations of the free energy, critical temperature $T_{c}$, magnetization $M$, and magnetic susceptibility $\chi$ as functions of the magnetic flux $\Phi$ through the hollow in a stack of mesoscopic superconducting cylinders are studied both analytically and numerically. The shape of these oscillations at low temperature $T$ and small level broadening $v$ is generally nonsinusoidal and has singularities that depend on the superconducting order parameter $\Delta$ and stacking sequence. The period of the oscillations is equal to the normal flux quantum $\Phi_{0}$. The harmonic amplitudes of the $\mathrm{AB}$ oscillations decrease exponentially if the diameter $2 R$ of the cylinders becomes greater than the coherence length. Further increase of $R$ results in a complete depression of the AB oscillations and the development of the parabolic Little-Parks (LP) oscillations of $T_{c}(\Phi)$, with half the period, $\Phi_{s}=\Phi_{0} / 2$. Therefore a crossover from the $\mathrm{AB}$ to $\mathrm{LP}$ oscillations takes place as the diameter $2 R$ is increased. It is shown that the temperature behavior of the magnetic susceptibility below the superconducting transition is $\chi \propto \exp \left(-T / T^{*}\right)$, where $T^{*}=\hbar v_{0} / 2 \pi^{2} R$. Such dependence of $\chi(T)$ has been observed recently in Ag wires coated with thin Nb layers in a weak external field [19] ( $v_{0}$ is the Fermi velocity, and $\hbar$ is Planck's constant).
\end{abstract}

PACS: 74.62.-c, 73.20.Dx

\section{Introduction}

Recently considerable attention has been devoted to studies of quantum oscillations in various artificially fabricated nanostructures subject to small external magnetic fields. In particular, different types of superconducting devices have been explored which exhibited oscillations of the critical temperature $T_{c}$ upon monotonic variation of the external magnetic field $H$. Such oscillations have been observed in a fractal networks [1,2], in Josephson junction arrays [3], and various mesoscopic loops [4-6]. All these experiments show diverse and basically nonsinusoidal oscillation patterns for the transition temperature $T_{c}(H)$ with the superconducting flux quantum $\Phi_{s}=\hbar c / 2 e$ as a fundamental period. It was established that such oscillations could be sufficiently well understood and described within the Ginzburg-Landau (GL) approach. The GL equation, when linearized in the order parameter $\Psi$, is formally identical to the Schrödinger equation for a doubly charged (in units of the electron charge $e$ ) particle in a magnetic field. Thus, the double charge of the Cooper pairs, $2 e$, is the physical reason standing behind the fact that quantity $\Phi_{s}$ determines the period of oscillations in the flux $\Phi$ for different nonsimply connected systems in the GL regime. The GL approach also proved to be successful in explanation of different known types of flux quantization in superconductors: the Little-Parks oscillations $[7,8]$, oscillations in superconducting fractal networks [9-11], and in Josephson junction arrays [12,13].

The Little-Parks (LP) oscillations of the critical temperature $T_{c}(H)$ have a parabolic shape and take place for samples whose sizes are of the order of the GL coherence length or greater. Oscillations as a function of flux in the GL regime, ignore the quantum phase interference phenomena due to the Aharonov-Bohm (AB) effect [14], which is normally present in the quasiparticle energy spectrum of nonsimply connected mesoscopic superconductors. The $\mathrm{AB}$ effect has numerous applications in different mesoscopic structures: conducting, insu- 
lating, magnetic, and strongly correlated, a review of which can be found in Refs. 15, 16. A general theoretical consideration of the $\mathrm{AB}$ effect in superconductors was given in the article [17], where a number of theorems have been formulated concerning hollow superconductors in a magnetic field.

The effect of flux quantization on the energy spectrum of excitations in a thin hollow superconducting cylinder and on the critical temperature $T_{c}(\Phi)$ was studied in [18]. It was shown in [18] that $T_{c}(\Phi)$ is an oscillatory function of the flux through the hollow $\Phi=H \pi R^{2}$ with the period $\Phi_{0}=2 \Phi_{s}(R$ is the inner radius of the cylinder). The authors proved the periodicity of the critical temperature, $T_{c}(\Phi)=T_{c}\left(\Phi+\Phi_{0}\right)$, and estimated the amplitude of oscillations as

$$
\frac{T_{c 0}-T_{c}}{T_{c}} \approx\left(\frac{\lambda_{B}}{R}\right)^{1 / 2} \exp \left(-2 \gamma R / \xi_{0}\right) .
$$

The explicit shape of oscillations was not found in [18]. The notation adopted in Eq. (1) is as follows: $\xi_{0}=\hbar v_{0} / \Delta \Delta(0)$ is the coherence length; $\lambda_{B}$ is the Bohr radius, and $\ln \gamma$ equals the Euler constant.

The purpose of present paper is to study (both analytically and numerically) the Aharonov-Bohm oscillations of the critical temperature $T_{c}(\Phi)$, magnetization $M(\Phi)$, and susceptibility $\chi$ in superconductors in more detail and to show their formal relation to the de Haas-van Alphen oscillations in superconductors - a very active area of research at the moment (see, for example, Refs. 25, 28, 29 and numerous references therein). To do this, we consider in what follows the $\mathrm{AB}$ oscillations of the free energy in a stack of weakly connected uniaxial hollow superconducting cylinders, which can be considered, for instance, as a model system for superconducting nanotubes [30]. We will show that the $T_{c}$ and the magnetic susceptibility $\chi$ in the $\mathrm{AB}$ regime are oscillating functions of the flux due to the quantities $\cos \left(2 \pi p \Phi / \Phi_{0}\right)$. For each integer $p$ the amplitudes of these oscillations depend on the electron level broadening $v=\hbar / \tau$, the order parameter $\Delta$, and the so-called stacking factor $I_{p}$, depending on $p$ in the very same fashion as for the de Haas-van Alphen $(d H v A)$ oscillations in layered superconductors [25]. We will show, in particular, that $\chi \propto \exp \left(-T / T^{*}\right)$, where $T^{*}=\hbar v_{0} / 2 \pi^{2} R$. This result exactly coincides with the experimental observation of the temperature behavior of the susceptibility in the $\mathrm{Ag} / \mathrm{Nb}$ wire bundle reported in Ref. 19. The stacking factor $I_{p}$ is a quantity which depends on the distribution of cylinders heights along the stack as well as the types of electrical contacts between them. It nontrivially modifies the amplitude of the $p$ th oscillation harmonic and can be determined by the relation [25]

$$
I_{p}=\int_{-\infty}^{\infty} g(\varepsilon) \exp \left(-i 2 \pi p \varepsilon / \varepsilon_{0}\right) d \varepsilon,
$$

where $g(\varepsilon)$ is a one-dimensional density of states related to the electron motion along the field (along the stack, in our case) and $\varepsilon_{0}$ is the typical energy separation between the energy levels in the problem. We will show in what follows that in the $\mathrm{AB}$ regime of a stack of mesoscopic cylinders $\varepsilon_{0}=\hbar^{2} k_{0} / M R$, where $k_{0}$ stands for the Fermi momentum and $M$ is the electron mass. In case of the de Haas-van Alphen oscillations in layered conductors [20,21] and superconductors [25] $\varepsilon_{0}=\hbar \Omega$, where $\Omega$ is the cyclotron frequency.

\section{The energy spectrum and the free energy}

Consider a stack of uniaxial thin hollow cylinders (or a tube) of inner radius $R$ and thickness $d<<R$, which is subject to an external magnetic field $H$ along the axis of the stack. The cylinders may be uniform in size, or their heights may vary periodically along the tube or be randomly distributed quantities. We assume also that the tunneling barriers for electron hopping between neighboring cylinders are weak enough that one can neglect the spatial variations of the superconducting order parameter along the stack (see Ref. 31 and the discussion in Sec. 4 in this connection).

Before considering the superconducting properties of the system in question, let us first determine its energy spectrum and the wave functions in the normal state. Taking then the $z$ axis of the cylindrical coordinates $(\rho, \varphi, z)$ to be parallel to the stack, and separating variables in the Schrödinger equation $H \Psi_{\varepsilon}=\varepsilon \Psi_{\varepsilon}$, we have

$$
\begin{gathered}
\Psi_{\varepsilon}(\mathbf{r})=\frac{1}{(2 \pi)^{1 / 2}} \mathrm{e}^{i n \varphi} f_{l}(\rho) \Psi_{\zeta m}(z) \\
\frac{1}{\rho} \frac{d}{d \rho}\left(\rho \frac{d f_{l}}{d \rho}\right)+\frac{\hbar^{2}}{2 M \rho^{2}}\left(n-\frac{\Phi(\rho)}{\Phi_{0}}\right)^{2} f_{l}(\rho)=\varepsilon_{n l} f_{l}(\rho) \\
\hat{H}_{z} \Psi_{\zeta m}(z)=\zeta_{m} \Psi_{\zeta m}(z) .
\end{gathered}
$$

Here $\rho=R+r$ is the radial coordinate $(0 \leq r \leq d<<R)$, and $\Phi(\rho)=H \pi \rho^{2}$ is the flux through a circle of radius $\rho$. The Schrödinger equation (5) describes the motion of an electron with 
energy $\zeta_{m} \equiv \varepsilon-\varepsilon_{n l}$ along the magnetic field (i.e., along the $z$ axis). Taking into account that in a thin cylinder $r / R<<1$, we find from Eq. (4) the approximate energy spectrum for electrons in the cylinder:

$$
\varepsilon_{n l}=\frac{\hbar^{2}}{2 M}\left[\frac{\pi^{2} l^{2}}{d^{2}}+\frac{1}{R^{2}}\left(n-\frac{\Phi}{\Phi_{0}}\right)^{2}\right]
$$

The appropriate radial wave function which satisfies the zero boundary conditions at the inner and outer surfaces of the cylinder, $f_{l}(R)=f_{l}(R+d)=0$, is given by

$$
f_{l}(\rho)=\sqrt{2 / d} \sin \left[\frac{\pi(\rho-R) l}{d}\right] .
$$

The quantum numbers in Eqs. (6) and (7) take the following values: $n=0, \pm 1, \pm 2, \ldots, l=0,1,2$, ... To calculate the quasiparticle energy of the system under study in the superconducting state, one has to address the Bogoliubov-de Gennes (BdG) equations:

$$
\begin{gathered}
\left(\hat{H}-E_{F}\right) u(\mathbf{r})+\Delta(\mathbf{r}) v(\mathbf{r})=E u(\mathbf{r}), \\
-\left(\hat{H}^{*}-E_{F}\right) v(\mathbf{r})+\Delta^{*}(\mathbf{r}) u(\mathbf{r})=E v(\mathbf{r}),
\end{gathered}
$$

where $E_{F}$ is the Fermi energy.

Since the thickness of cylinders is much less than the BCS coherence length $\xi$, the order parameter $\Delta$ does not depend on the coordinate $\mathbf{r}$. Taking this into consideration and expanding the $u-v$ functions in the basis of Eq. (3),

$$
u(\mathbf{r})=\sum_{\varepsilon} u_{\varepsilon} \Psi_{\varepsilon}(\mathbf{r}), \quad v(\mathbf{r})=\sum_{\varepsilon} v_{\varepsilon} \Psi_{\varepsilon}(\mathbf{r}),
$$

we find the quasiparticle energy from the $\mathrm{BdG}$ equations (8):

$$
E_{n l}=\sqrt{\left(\varepsilon_{n l}-E_{F}\right)^{2}+\Delta^{2}} .
$$

Proceeding then in a standard fashion, we arrive at the spectral density for quasiparticles with a small damping, $v=\hbar / \tau$, as a sum of two Lorentzians [22],

$$
\begin{aligned}
\rho_{n}\left(\xi_{m}, \omega\right) & =\frac{1}{\pi} u_{n}^{2} \frac{v}{v^{2}+\left(\omega+E_{n}\left(\xi_{m}\right)\right)^{2}}+ \\
+v_{n}^{2} & \frac{v}{v^{2}+\left(\omega+E_{n}\left(\xi_{m}\right)\right)^{2}} .
\end{aligned}
$$

The quantities $u_{n}^{2}$ and $v_{n}^{2}$ here stand for the coherence factors:

$$
u_{n}^{2}\left(\xi_{m}\right)=\frac{1}{2}\left(1+\frac{\varepsilon_{n}\left(\xi_{m}\right)}{E_{n}\left(\xi_{m}\right)}\right), v_{n}^{2}\left(\xi_{m}\right)=\frac{1}{2}\left(1-\frac{\varepsilon_{n}\left(\xi_{m}\right)}{E_{n}\left(\xi_{m}\right)}\right)
$$

We put for simplicity $l=1$, so that the quasiparticle energies in the normal and superconducting states become, respectively,

$$
\varepsilon_{n}\left(\xi_{m}\right)=\varepsilon(R)\left(n-\frac{\Phi}{\Phi_{0}}\right)^{2}+\xi_{m}-\mu
$$

and

$$
E_{n}\left(\xi_{m}\right)=\sqrt{\varepsilon_{n}\left(\xi_{m}\right)^{2}+\Delta^{2}},
$$

where $\mu$ and $\varepsilon(R)$ are determined by the relations

$$
\mu=E_{F}-\frac{\hbar^{2} \pi^{2}}{2 M d^{2}}, \varepsilon(R)=\frac{\hbar^{2}}{2 M R^{2}} .
$$

Once the spectral density is found, the free energy of a superconductor can then be calculated with the help of the formula [23]

$$
\begin{gathered}
F=\int d \mathbf{r} \frac{|\Delta(\mathbf{r})|^{2}}{\lambda}- \\
-2 T \sum_{n, m} \int_{-\infty}^{\infty} d \omega \rho_{n}\left(\xi_{m}, \omega\right) \ln \left[2 \cosh \left(\frac{\omega}{2 T}\right)\right],
\end{gathered}
$$

where $\lambda$ is the BCS coupling constant. Using the integral representation for the logarithm in Eq. (16) [24]

$$
\ln \left[\cosh \left(\frac{\omega}{2 T}\right)\right]=\int_{-\infty}^{\infty} d z \frac{1-\cos \omega z}{z \sinh \pi T z}
$$

and the Poisson summation rule

$$
\sum_{n_{0}}^{\infty} \chi(n)=\int_{-\infty}^{\infty} \chi(n) d n+2 \operatorname{Re} \sum_{p=1}^{\infty} \int_{a}^{\infty} \chi(n) \mathrm{e}^{i 2 \pi n p} d n
$$

where $n_{0}-1<a<n_{0}$, we find the oscillatory part of the free energy in the form

$$
F_{\text {osc }}=\frac{4 \pi T}{\varepsilon_{0}} \sum_{p=1}^{\infty} Q_{p} \Psi_{p}(T, \Delta, v) \cos \left(2 \pi p \frac{\Phi}{\Phi_{0}}\right)
$$


It is important to note that nonoscillatory part of the free energy, determined by the first integral term in Eq. (18), does not depend on the flux, because $\varepsilon_{n}\left(\xi_{m}\right)$ in Eq. (13) depends on $n$ through the combination $n-\Phi / \Phi_{0}$. Two factors $\Psi_{p}(T, \Delta, v)$ and $Q_{p}$ have been introduced in Eq. (19):

$$
\Psi_{p}(T, \Delta, v)=\int_{-\infty}^{\infty} d z G_{p}(z) \frac{\mathrm{e}^{-v|z|}}{z \sinh (\pi T z)},
$$

and

$$
Q_{p}=\operatorname{Re}\left[I_{p} \exp \left(i 2 \pi p R k_{0}\right)\right] .
$$

The function $G_{p}(z)$ is determined by the integral

$$
G_{p}(z)=\int_{-\infty}^{\infty} d n \cos \left(z \sqrt{\left(\varepsilon_{0} n\right)^{2}+\Delta^{2}}\right) \cos (2 \pi n p) .
$$

Thus the factor $\Psi_{p}(T, \Delta, v)$, given by Eq. (20), describes the damping of the $\mathrm{AB}$ oscillations of the free energy due to the BCS gap $\Delta$ and energy level broadening $v$. The factor $Q_{p}$ in Eq. (20) modulates the $p$ th harmonic through the stacking factor $I_{p}$, which has already been determined by Eq. (2). It depends on the one-dimensional density of states associated with the electron motion along the stack

$$
g(\varepsilon)=\sum_{m} \delta\left(\varepsilon-\xi_{m}\right)
$$

The factor analogous to the $I_{p}$ was introduced earlier in the theory of de Haas-van Alphen oscillations in layered conductors [20,21] and superconductors [25].

The integral in Eq. (22) can be calculated exactly to yield [24]

$G_{p}(z)=\frac{\pi}{\varepsilon_{0}}\left[\delta\left(z-z_{p}\right)+\theta\left(z-z_{p}\right) \frac{\partial}{\partial z} J_{0}\left(\Delta \sqrt{z^{2}-z_{p}^{2}}\right)\right]$,

where $\theta(z)$ is the Heaviside step function; $J_{0}(z)$ stands for the Bessel function; and $z_{p}=2 \pi p / \varepsilon_{0}$. Now substituting Eq. (24) into Eq. (20), we have

$$
\begin{gathered}
\Psi_{p}(T, \Delta, v)= \\
=\Psi\left(z_{p}, v\right)+\int_{z_{p}}^{\infty} d z \Psi(z, v) \frac{\partial}{\partial z} J_{0}\left(\Delta \sqrt{z^{2}-z_{p}^{2}}\right),
\end{gathered}
$$

where

$$
\Psi(z, v)=\frac{\mathrm{e}^{-v|z|}}{z \sinh (\pi T z)} .
$$

The second term in (25) vanishes when $\Delta=0$, so that $\Psi\left(z_{p}, v\right)$ is the factor which determines the amplitudes of the $\mathrm{AB}$ oscillations in the stack in the normal state. The dependence of $F_{\text {osc }}$ on the oscillating factors $\cos \left(2 \pi p \Phi / \Phi_{0}\right)$ is a manifestation of the AB effect $[16,27]$ in mesoscopic systems. Amplitudes $Q_{p}$ and $\Psi_{p}$ formally are much the same as those appearing in the de Haas-van Alphen effect in layered superconductors in the vortex state below the upper critical magnetic field [25]. The latter is because in both cases oscillation effects arise due to the quantization of the energy spectrum in superconductors. The principal difference in the field dependence of $F_{\text {osc }}(H)$ in the de Haas-van Alphen and Aharonov-Bohm cases appears because of the different nature of the quantization. In case of the de Haas-van Alphen effect the strong external magnetic field itself is a quantizing factor, which yields a discrete Landau spectrum responsible for the periodicity of the free energy $F_{\text {osc }}(H)$ in the inverse magnetic field $1 / H$. In case of the $\mathrm{AB}$ problem under study, the energy levels $\varepsilon_{n}\left(\xi_{m}\right)$ of Eq. (13) appear due to the size quantization in the mesoscopic sample, and the flux $\Phi \propto H$ enters the discrete spectrum $\varepsilon_{n}\left(\xi_{m}\right)$ as a parameter thereby making the free energy $F_{\text {osc }}(\Phi)$ in Eq. (24) a periodic function of the field itself. Physically, the dependence on $\Phi$ in the $\mathrm{AB}$ systems should vanish together with the size quantization in the bulk limit. In the next Sections we will see in detail how the $\mathrm{AB}$ oscillations manifest themselves in the mesoscopic tube under consideration, both in the normal and superconducting states.

\section{The critical temperature oscillations and dimensional crossover}

Near the critical temperature one can expand the free energy in powers of $\Delta^{2}$. Using Eqs. (16), (18), and (19), we have

$$
F_{S}-F_{N} \approx
$$

$\approx \Delta^{2}\left[\alpha(T)-\frac{2}{\varepsilon_{0}} \sum_{p=1}^{\infty} Q_{p} N_{p}(T, v) \cos \left(2 \pi p \frac{\Phi}{\Phi_{0}}\right)\right]+\ldots$, 
where $\alpha(T)$ is the first coefficient of the standard Ginzburg-Landau expansion. In the case of a superconductor of small dimensions it can be found directly from the free energy determined by Eq. (16) (see Ref. 26 for details):

$$
\alpha(T)=N(0)\left[\ln \left(\frac{T}{T_{c}^{0}}\right)+\Psi\left(\frac{1}{2}+\frac{v}{2 \pi T}\right)-\Psi\left(\frac{1}{2}\right)\right] .
$$

Here $T_{c}^{0}=T_{c}^{0}(R)$ is the critical temperature of a small cylinder of radius $R ; \Psi$ stands for the digamma function; $N(0)$ is the density of states. The factor $N_{p}(T, v)$ entering the free energy expansion in Eq. (27) is determined by the integral

$$
N_{p}(T, v)=\int_{a_{p}}^{\infty} d z \frac{\exp (-b z)}{\sinh z}
$$

where $b=\mathrm{v} / \pi T$ and $a_{p}=2 \pi R p / \xi(T)$. The coherence length depends on the Fermi velocity $v_{0}$ and the temperature according to the relation $\xi(T)=\hbar v_{0} / \pi T$.

The critical temperature of the stack can be determined from the Ginzburg-Landau expansion by equating to zero the term in the square brackets in Eq. (28). Taking into account a smallness of the oscillatory correction to the critical temperature $T_{c}^{*}(R)$ of a cylinder due to the Aharonov-Bohm effect, we have

$$
\begin{gathered}
T_{c}(\Phi)=T_{c}^{*}(R)- \\
-\frac{2 T_{c}^{0}(R)}{N(0) \varepsilon_{0}} \sum_{p=1}^{\infty} Q_{p} N_{p}\left[T_{c}^{0}(R), v\right] \cos \left(2 \pi p \frac{\Phi}{\Phi_{0}}\right) .
\end{gathered}
$$

Here $T_{c}^{*}(R)=T_{c}^{0}(R)-\pi v / 8$ is the transition temperature of the cylinder, taking into account a small depression due to the size-quantization level broadening. Thus the critical temperature $T_{c}(\Phi)$ oscillates in the direct field with a period equal to the normal flux quantum $\Phi_{0}$. The amplitudes of harmonics in Eq. (30) depend on two factors $Q_{p}$ and $N_{p}$, of which $Q_{p}$ is proportional to the stacking factor $I_{p}$. This factor depends on the way which cylinders are stacked into a tube and on the type of electrical contact between them. Consider, as a specific example, a model in which identical cylinders are stacked periodically into a tube and the tunnel junctions between them are determined by the hopping integral $\sigma$. Such a model has recently found support in experiments on carbon nanotubes [31]. The corresponding density of states, associ- ated with the electron transport along the tube in this model, is given by the relation $g(\varepsilon)=1 / \pi\left(4 \sigma^{2}-\varepsilon^{2}\right)^{1 / 2}$. Integration of Eq. (2) with this $g(\varepsilon)$ yields $I_{p}=J_{0}\left(4 \pi \sigma p / \varepsilon_{0}\right)$, so that the factor $Q_{p}$ becomes

$$
Q_{p}=2 J_{0}\left(\frac{4 \pi \sigma p}{\varepsilon_{0}}\right) \cos \left(2 \pi p R k_{0}\right)
$$

( $J_{0}(x)$ is the Bessel function). The case of a single cylinder corresponds to the prohibition of electron tunneling between the adjacent cylinders, i.e., $\sigma=0$. The density of states in this limit is given by $g(\varepsilon)=\delta(\varepsilon)$, which implies that $I_{p}$ equals unity and $Q_{p}=2 \cos \left(2 \pi p R k_{0}\right)$. Having at hand Eqs. (29) and (31), one can calculate the sum in Eq. (30), which determines the shape of the oscillations of the critical temperature $\delta T_{c}(\Phi) \equiv\left(T_{c}(\Phi)-T_{c}^{*}(R)\right) / T_{c}^{0}(R)$ in units of the quantity $N(0) \varepsilon_{0}$. The results of the numerical calculations of $\delta T_{c}(\Phi)$ for different values of the parameters $a=2 \pi^{2} T_{c}^{0}(R) / \varepsilon_{0}$ and $b=v / \pi T_{c}^{0}(R)$ are shown in Fig. 1. We see that in general the shape of the Aharonov-Bohm oscillations of the transition temperature in the superconducting tube is not of the simple cosine form $\cos \left(2 \pi \Phi / \Phi_{0}\right)$ because of the contributions from the higher harmonics $(p=2,3, \ldots)$. It takes the harmonic sinelike form only for sufficiently large $a$ and $b$ as one can see in Fig. 1,e. These quantities depend on $T_{c}^{0}(R)$ and the energy level broadening $v$, which therefore act as factors damping the amplitudes and smearing the fine structure of these oscillations. The value of the hopping integral also strongly affects the shape and amplitudes of the oscillations, as one can tell by comparing Fig. 1, $a$ and Fig. 1, $b$. The enhancement of $\sigma$ changes the oscillation pattern and strongly depresses the amplitudes.

The temperature and broadening $v$ influence the amplitude of the $p$ th harmonic through the factor $N_{p}(T, v)$, which decreases rapidly as the parameters $b$ and $a_{p}$ increase. In the case when $2 \pi R>>\xi(T)$, this factor becomes an exponential function

$$
N_{p}(T, v) \approx \frac{2}{1+b} \exp \left[-\left(\frac{T}{T^{*}}\right) p\right] \exp \left(-\frac{v}{\pi T^{*}}\right)
$$

depending on some characteristic temperature $T^{*}=$ $=\hbar v_{0} / 2 \pi^{2} R$, which is a decreasing function of the radius $R$. It follows then from Eqs. (30) and (32) that the quantum $\mathrm{AB}$ oscillations of the critical temperature decrease exponentially with $R$ if $2 \pi R>>\xi\left(T_{c}^{0}\right)$. On the other hand, when $R$ exceeds the Ginzburg-Landau coherence length $\xi_{G L}\left(T_{c}^{0}\right)$ 


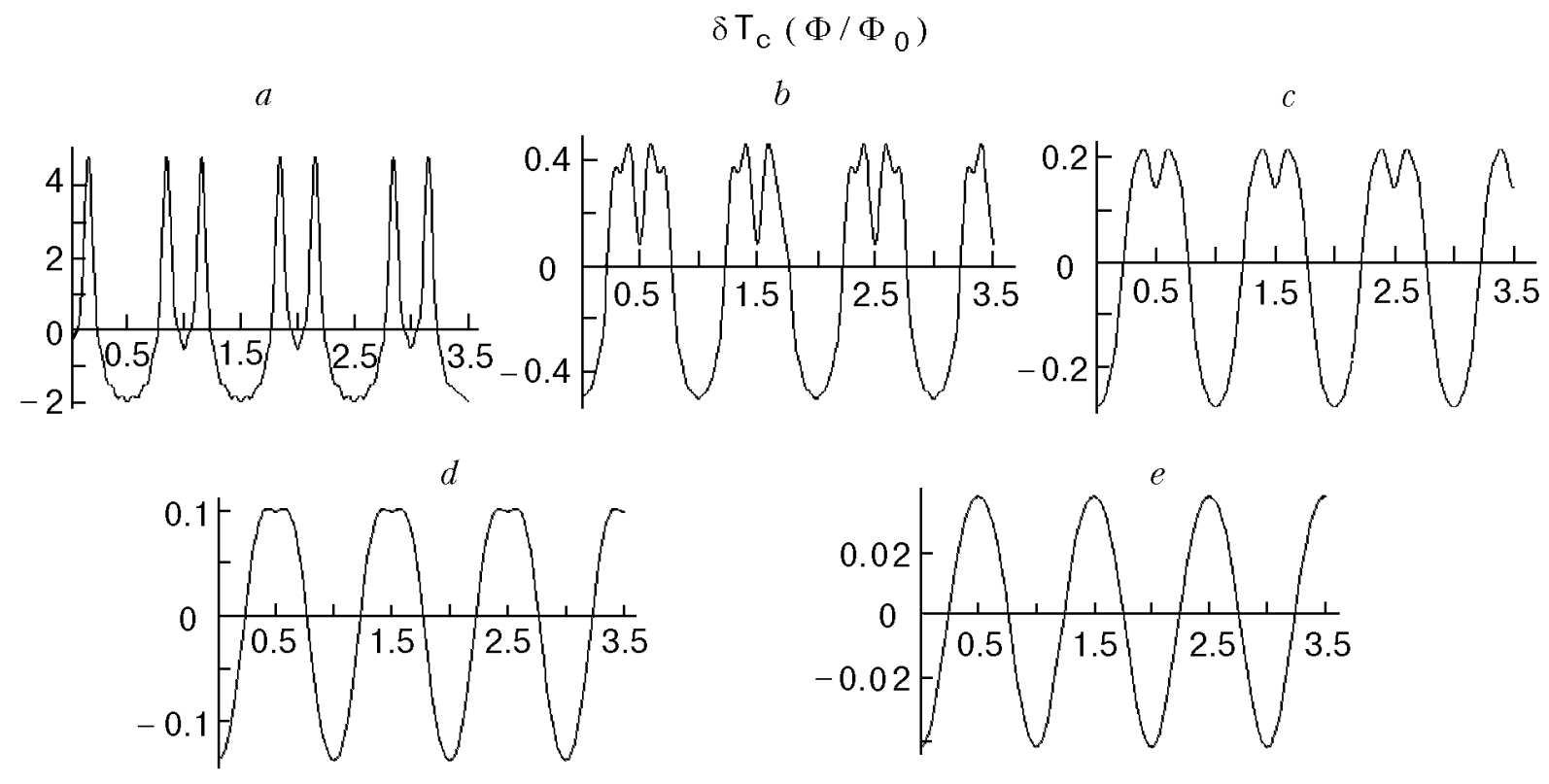

Fig. 1. The Aharonov-Bohm oscillations of the critical temperature of a stack of hollow superconducting cylinders $\delta T_{c}\left(\Phi / \Phi_{0}\right) \equiv\left(T_{c}(\Phi)-T_{c}^{*}(R)\right) / T_{c}^{0}(R)$ (see Eq. (30)) in units of the quantity $N(0) \varepsilon_{0}$ ( $\Phi$ is the flux through a hollow, and $\Phi_{0}$ denotes the flux quantum). The numerical calculations of $\delta T_{c}(\Phi)$ in Figs. 1,a-1e are done for different values of the parameters $a=2 \pi^{2} T_{c}^{0}(R) / \varepsilon_{0}, b=\mathrm{v} / \pi T_{c}^{0}(R), c=4 \pi \sigma / \varepsilon_{0}$, and $R k_{0}=1000: a=0.1, b=1, c=0.1$ (Fig. $\left.1, a\right) ; a=0.1, b=1, c=10($ Fig. $1, b) ; a=$ $0.25, b=1, c=10$ (Fig. 1,c); $a=0.5, b=1, c=10$ (Fig. 1,d); $a=1, b=1, c=10$ (Fig. 1,e).

(i.e., in the GL regime), the gradient term in the free energy expansion should be added to the righthand side of Eq. (27):

$$
\frac{1}{2 m}\left|\left(\frac{\hbar}{i} \nabla-\frac{2 e \mathbf{A}}{c}\right) \Psi\right|^{2}
$$

The Ginzburg-Landau order parameter $\Psi$ in Eq. (33) is proportional to $\Delta$ [32]: $\Psi=\gamma^{1 / 2} \Delta$, where $\gamma^{2}=7 \zeta(3) m v_{0}^{2} N(0) / 2 \pi^{2} T_{c}^{0}$ for clean superconductors and $\gamma^{2}=\pi m v_{0} N(0) l / 12 \hbar T_{c}^{0}$ for dirty superconductors. Here $v_{0}$ is the Fermi velocity, and $\zeta(3)$ is the zeta function, $l=\hbar v_{0} / \mathrm{v}$. The fluxoid quantization in a thin superconducting cylinder makes the supercurrent velocity $v_{s}=(\hbar / m R) \min \left|\left(n-\Phi / \Phi_{s}\right)^{2}\right|$ an oscillatory function of the ratio $\Phi / \Phi_{s}$ which is a manifestation of the Little-Parks effect [7,8] ( $n$ is an integer). Thus the free energy expansion of Eq. (27) in the GL regime takes the form

$$
F_{S}-F_{N} \approx \Psi^{2}\left[\gamma\left\{\alpha(T)-\frac{2}{\varepsilon_{0}} \sum_{p=1}^{\infty} Q_{p} N_{p}(T, v) \cos \left(2 \pi p \frac{\Phi}{\Phi_{0}}\right)\right\}+\frac{m v_{s}^{2}}{2}\right]+\ldots
$$

We see that two physically different regimes should be distinguished. In the mesoscopic regime, $2 \pi R \leq \xi(T)$, the free energy given by Eq. (27) oscillates with period $\Phi_{0}$ on account of the $\mathrm{AB}$ effect. In the Ginzburg-Landau regime, $2 \pi R>>\xi\left(T_{c}^{0}\right)$, the harmonic oscillations given by the sum over $p$ in Eq. (34) vanish, and the period of oscillations becomes equal to the superconducting flux quantum $\Phi_{s}=\Phi_{0} / 2$. In this case the oscillations are completely determined by the supercurrent term $m v_{s}^{2} / 2$, as in the case of the conventional Little-Parks effect. The critical temperature oscillates in this regime according to the standard Little-Parks equation

$$
T_{c}^{0}(\Phi)=T_{c}^{*}(R)+0.73 \frac{\xi_{0} l}{R^{2}} T_{0}(R) \min \left[\left(n-\frac{\Phi}{\Phi_{s}}\right)^{2}\right]
$$

Thus an increase of $R$ results in a crossover from harmonic oscillations of the critical temperature $T_{c}(\Phi)$ with period $\Phi_{0}$ in the Aharonov-Bohm regime (see Eq. (30)) to the parabolic Little-Parks 
oscillations with period $\Phi_{s}$ in the Ginzburg-Landau regime described by Eq. (35).

\section{Magnetization, persistent current, and susceptibility oscillations}

Having at hand equations for the free energy, one can calculate the magnetization $M$ and the susceptibility $\chi$ by taking derivatives:

$$
M=-\frac{\partial F}{\partial H}, \quad \chi=\frac{\partial M}{\partial H} .
$$

Consider first the magnetization. In the $\mathrm{AB}$ regime $H$ enters the free energy, as one can see from Eqs. (24) and (28), through a strongly oscillatory function $\cos \left(2 \pi p \Phi / \Phi_{0}\right)$, so that derivation in Eq. (36) is trivial and yields

$M_{\mathrm{osc}}=-\frac{4 \pi T}{\varepsilon_{0}}\left(\frac{2 \pi S}{\Phi_{0}}\right)_{p=1}^{\infty} Q_{p} \Psi_{p}(T, \Delta, v) p \sin \left(2 \pi p \frac{\Phi}{\Phi_{0}}\right)$

It is instructive to begin the analysis of the shape of the function $M_{\mathrm{osc}}(\Phi)$ with consideration of the limiting case $\Delta=\sigma=v=T=0$, since the sum in Eq. (37) in this case can be calculated analytically. Setting to zero these parameters in Eq. (37), we have

$$
M_{\mathrm{osc}}(\Phi)=M_{\mathrm{osc}}^{+}+M_{\mathrm{osc}}^{-}
$$

where

$$
M_{\mathrm{osc}}^{ \pm}=\frac{2 S \varepsilon_{0}}{\pi \Phi_{0}} \sum_{p=1}^{\infty} \frac{(-1)^{p}}{p} \sin \left[2 \pi p\left(\frac{\Phi}{\Phi_{0}} \pm R k_{0}+\frac{1}{2}\right)\right]
$$

The sum in Eq. (39) is exactly a saw-tooth function of its argument in the parenthesis. Therefore the magnetization $M_{\text {osc }}(\Phi)$ of a single cylinder $(\sigma=0)$ at zero temperature and level broadening $v$ is a sum of two saw-tooth profiles, as is shown in Fig. 2, $a$. The persistent current and the corresponding magnetization function $M_{\text {osc }}(\Phi)$ of a thin normal ring (first obtained by Kulik [27]) were studied in the paper [33] and later discussed in detail in a review article [34]. Nonetheless, as one can see from Eq. (37), the magnetization of the tube is strongly affected by the additional factor $Q_{p}(\sigma)$. The role of the stacking factor $Q_{p}(\sigma)$ in the shape of $M_{\text {osc }}(\Phi)$ is nontrivial and is illustrated by the relative transfigurations of the oscillation patterns shown in
Figs. 2, $a$ and 2,b. Increasing the temperature and level broadening damp the amplitudes of the harmonics, making the form of $M_{\text {osc }}(\Phi)$ close to sinelike, as one can see in Fig. 2,c. The shape of the magnetization oscillations in the superconducting tube calculated on the basis of Eq. (37) for different values of the parameters $\Delta, \sigma, v$, and $T$ is shown in Fig. 3. The magnetization of the stack of cylinders is inherently related to the so-called persistent current $I=c M / S\left(S=\pi R^{2}\right)$. This current flows around the hollow and thereby creates the magnetization. Therefore oscillations of the magnetization as a function of flux also imply oscillations of the persistent current, with the amplitude $I_{\text {osc }}(\Phi)=c M_{\text {osc }}(\Phi) / S$. The oscillations of the susceptibility can be calculated by taking the derivative $\chi_{\text {osc }}(\Phi)=\partial M_{\text {osc }}(\Phi) / \partial H$. In the normal state, under the conditions $\Delta=\sigma=v=$ $=T=0$, the magnetization function $M_{\mathrm{osc}}(\Phi)$ is given by the saw-tooth function of Eq. (38) and is
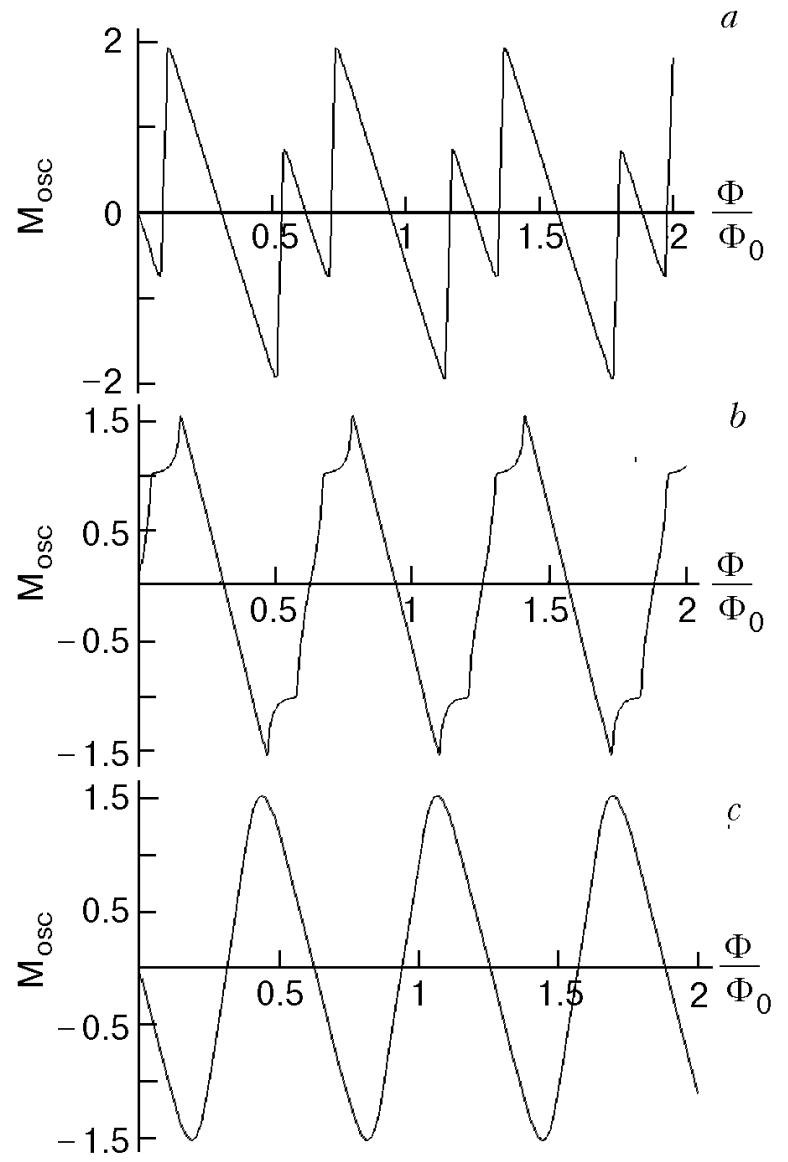

Fig. 2. The Aharonov-Bohm oscillations of the magnetization of a stack of hollow normal cylinders $M_{\text {osc }}\left(\Phi / \Phi_{0}\right.$ ) (given by Eq. (37) with $\Delta=0$ ) in units of the quantity $M(0)=2 \varepsilon_{0} S / \Phi_{0}$. The numerical calculations of $M_{\mathrm{osc}}\left(\Phi / \Phi_{0}\right)$ in Figs. 1, $a-1 c$ are done for different values of the parameters $a=2 \pi^{2} T_{c}^{0}(R) / \varepsilon_{0}$, $b=\mathrm{v} / \pi T_{c}^{0}(R), c=4 \pi \sigma / \varepsilon_{0}$, and $R k_{0}: a=0.01, b=0.01, c=0.1$, $R k_{0}=1000$ (Fig. 1,a); $a=0.01, b=0.01, c=1, R k_{0}=100$ (Fig. 1,b); $a=0.01, b=0.01, c=0.1, R k_{0}=100$ (Fig. 1,c). 
$a$
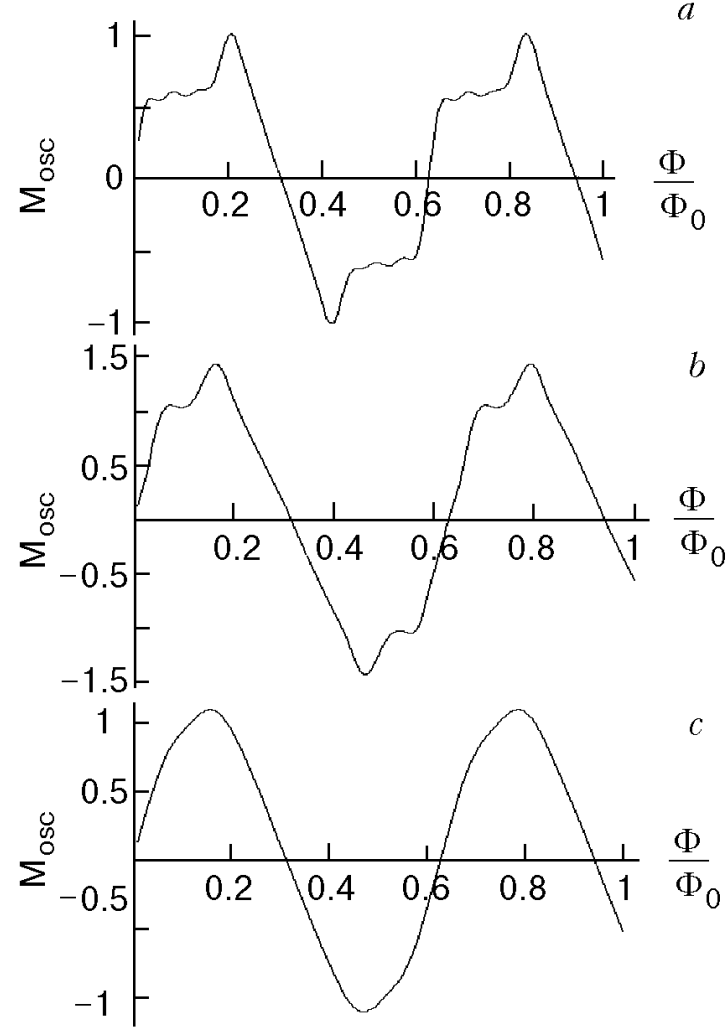

Fig. 3. The Aharonov-Bohm oscillations of the magnetization of a stack of hollow superconducting cylinders $M_{\text {osc }}\left(\Phi / \Phi_{0}\right)$ (see Eq. (37)) in units of the quantity $M(0)=2 \varepsilon_{0} S / \Phi_{0}$. The numerical calculations of $M_{\text {osc }}\left(\Phi / \Phi_{0}\right)$ in Figs. 1, $a-1, c$ are done for different values of the parameters $a=2 \pi^{2} T_{c}^{0}(R) / \varepsilon_{0}$, $b=v / \pi T_{c}^{0}(R), c=4 \pi \sigma / \varepsilon_{0}, d=2 \pi \Delta / \varepsilon_{0}$, and $R k_{0}: a=0.01$, $b=0.01, c=1, d=0.05, R k_{0}=1000$ (Fig. 1,a); $a=0.01, b=$ $=0.01, c=1, R k_{0}=100, d=0.05$ (Fig. 1,b); $a=0.01, b=0.5$, $c=1, d=0.5, R k_{0}=1000($ Fig. $1, c)$.

shown in Fig. 2,a. Thus $\chi_{\mathrm{osc}}(\Phi)$ in this case would have delta-function peaks at the jumps of the sawtooth and a negative constant value between them. Temperature and $v$, as well as $\Delta$ and $\sigma$, smear the singular features in $M_{\mathrm{osc}}(\Phi)$, as one can see in Fig. 2, $a$ and Fig. 3,c, making the $\chi_{\text {osc }}(\Phi)$ a harmoniclike function too. Near the transition temperature, provided that it is high enough that the higher harmonics can be neglected, we have from Eq. (27) a simple analytical expression for the susceptibility:

$$
\chi \approx \frac{2}{\varepsilon_{0}}\left(\frac{2 \pi S \Delta}{\Phi_{0}}\right)^{2} Q_{1} N_{1}(T, v) \cos \left(2 \pi \frac{\Phi}{\Phi_{0}}\right)
$$

According to Eq. (32) the temperature behavior of the susceptibility near $T_{c}$ is

$$
\chi \propto \exp \left(-\frac{T}{T^{*}}\right)
$$

Such a temperature dependence of the magnetic susceptibility has been found recently in experiments on a bundle of electrically isolated $\mathrm{Ag} / \mathrm{Nb}$ wires [19]. Every wire in the bundle was a very clean Ag cylinder coated with thin superconducting $\mathrm{Nb}$ layer. The authors of Ref. 19 also observed a crossover in the temperature behavior of the susceptibility which doubles the ratio $T / T^{*} \rightarrow 2 T / T^{*}$ in the exponent of $\chi(T)$ when $T<T^{*}$. We can relate this phenomenon to the fact that the quantity $\xi(T)=\hbar v_{0} / \pi T$ has a double meaning in the problem in question. First, it is the coherence length that determines the amplitudes of the $\mathrm{AB}$ oscillations. Second, the length $\xi_{N}(T)=\hbar v_{0} / \pi T$ is known to be the length that determines the spatial scale of the proximity effect. In the context of our analysis this means that at the temperature $T=T^{*}$ the Ag cylinder became completely superconducting due to the proximity with superconducting $\mathrm{Nb}$ coating, since at this temperature $\xi_{N}\left(T^{*}\right)=2 \pi R$. As a result, the flux is expelled from the interior of the wire, and the cylinder can no longer trap the flux in the $\mathrm{Ag}$ core. Therefore the crossover observed in the $\mathrm{Ag} / \mathrm{Nb}$ cylinders may be considered as a consequence of the crossover in the topology of the system as the temperature decreases: «hollow» cylinders with a flux trapped inside the normal Ag core (for temperatures $T>T^{*}$ ) became completely superconducting when $T$ becomes less than $T^{*}$ and expel the flux from the Ag core.

\section{Conclusions}

The original $\mathrm{AB}$ effect was formulated in [14] as a gedanken experiment in which the interference pattern of two electrons moving around the solenoid in the absence of a magnetic field depends on the flux through the solenoid. This theoretical prediction was soon confirmed in a real experiment done by Chambers [35]. Later on, different manifestations of the $\mathrm{AB}$ effect in solids have been studied and many examples were found in which various physical properties exhibit oscillations as functions of the flux with period $\Phi_{0}$ (many references on the subject contain review articles $[15,16,34])$. A theory of the $\mathrm{AB}$ oscillations of the free energy in a metal ring and the related problem of the persistent currents in such systems was first considered in the paper [27] (see also Ref. 36 in this connection) and was later generalized to the case of superconducting ring in Ref. 18, where Aharonov-Bohm oscillations of the transition temperature $T_{c}(\Phi)$ have been predicted. The theoretical consideration given in this work develops further the results of the papers $[18,27]$. For this purpose, we have studied the free 
energy of a stack of superconducting cylinders in external magnetic field within the microscopic BCS model and have calculated analytically the oscillatory part of the free energy (see Eq. (19)). We have also found explicit expressions for the $\mathrm{AB}$ oscillations of the critical temperature $T_{c}(\Phi)$ (Eq. (30)), the oscillatory part of the magnetization (Eqs. (37)-(39)), and the susceptibility $\chi(T)$ near the transition temperature (Eq. (40)). The oscillatory dependence of all above quantities on the flux $\Phi$ with the fundamental period $\Phi_{0}$ stems, in the long run, from the gauge invariance of the theory of nonsimply connected solids. In our particular case this flux dependence appears due to the quasiparticle energy given by Eq. (13). The results of a numerical analysis of these oscillations is shown in Figs. 1-3. These pictures demonstrate strongly nonsinusoidal oscillating patterns for the functions $T_{c}(\Phi)$ and $M_{\text {osc }}(\Phi)$ in the case of low temperatures and small level broadening. Such nonsinusoidal behavior of the oscillations is caused by the small decrease of the harmonic amplitudes with their number $p$ in this case. Increasing the temperature and $v$ as well as $\Delta$ damps the amplitudes of the harmonics and smears the singularities in the oscillation patterns, making them close to sinelike or cosinelike in shape. The way in which the cylinders are stacked into a tube also strongly affects the oscillation patterns through the factor $Q_{p}$ of Eq. (21). This factor in turn depends on the stacking factor $I_{p}$ given by Eq. (2) and determined by the density of states $g(\varepsilon)$ related to electron motion across the stack (along the tube). The dependence of the oscillation pattern on the hopping integral $\sigma$ between cylinders in a stack in a model described by the factor of Eq. (31) is rather sophisticated and can be seen from a comparison of Figs. 2, $a$ and 2,b. The Aharonov-Bohm effect has been observed recently in circular carbon nanotubes [30]. It was also shown that structural disorder effectively breaks carbon nanotubes into a series of cylinders (or dots) separated by tunneling barriers. The transmission probability between the cylinders was estimated to vary within the limits $0.001-0.1$ [31]. Thus nanotubes as well as coated wires are good candidates for the physical realization of the model adopted in this paper.

Being quantum in nature, the $\mathrm{AB}$ oscillations of the critical temperature $T_{c}(\Phi)$ vanish exponentially when $R$ is increased to values of the order of the GL coherence length. Further increase of $R$ results in a dimensional crossover from the $\mathrm{AB}$ oscillations to the Little-Parks ones. The LP oscillations have half as large a period in the flux, $\Phi_{s}=\Phi_{0} / 2$, and have the shape of periodic parabolas (see Eq. (35)). The LP oscillations take place in the GL regime $R \geq \xi_{G L}(T)$, in which the quantum size effects are irrelevant and quantum $\mathrm{AB}$ oscillations are suppressed. The LP oscillations arise due to externalfield-induced oscillations of the superconducting current of the Cooper pairs around a hollow in the cylinder. Since the Cooper pairs carry double electronic charge, the period of these oscillations in the flux becomes half as large as for the quantum $\mathrm{AB}$ oscillations. We also found that near the transition temperature the magnetic susceptibility $\chi(T)$ of the superconducting cylinder is given by Eq. (40), which, through the factor of Eq. (41), displays the nontrivial exponential temperature dependence observed in $\mathrm{Ag} / \mathrm{Nb}$ wires [19].

In conclusion, we would like to stress yet another important point of our consideration. The physics behind the quantum $\mathrm{AB}$ oscillations in superconductors, in essence, is very much similar to that which governs the dHvA oscillations in the vortex state. In spite of the fact that the $A B$ oscillations are periodic in the direct field, the damping factors $\Psi_{p}$ and $Q_{p}$ entering Eq. (19) are akin to the appropriate factors in the dHvA oscillations in superconductors [25]. For example, the factor $\Psi_{p}$ determined by Eq. (25), after the substitution $\varepsilon_{0}=\hbar \Omega$, becomes identical to the appropriate factor in the dHvA oscillations describing the damping of the $p$ th amplitude due to the superconducting gap $\Delta(T)$. It is impossible to single out experimentally the contribution of $\Delta$ to the damping of the dHvA oscillations in superconductors because of the spatial modulation of the order parameter $\Delta(\mathbf{r})$ caused by the vortices [28]. Since there are no vortices in the $A B$ system under consideration, the comparative experimental studies of the damping of the $\mathrm{AB}$ and dHvA oscillations can provide the information necessary for separation of the «vortex matter» contribution to the damping of the dHvA oscillations in superconductors in the mixed state.

\section{Acknowledgments}

The results of this work have been discussed with my untimely deceased friend Alexander Semenovich Rozhavsky, to whom I would like to express my deepest feelings of gratitude. I would also like to thank Il'ya Krive for helpful comments on the manuscript and for useful conversations. 
19. R. Frassanito, P. Visani, M. Niderost, A. C. Mota, P. Smeibidl, K. Swietca, W. Wendler, and F. Pobell, Czech. J. Phys. 46, 2317 (1996).

Lett. 56, 2280 (1986).

2. A. Gerber and G. Deucher, Phys. Rev. Lett. 64, 1585 (1990).

3. H. R. Shea and M. Tinkham, Phys. Rev. Lett. 79, 2324 (1997).

4. V. V. Moshchalkov, L. Giedu, C. Strunk, R. Jonckheere, X. Qui, C. Van Haesendonck, and Y. Bruynseraede, Nature 373, 319 (1995).

5. C. Strunk, V. Bruyndocx, V. V. Moshchalkov, C. Van Haesendonck, and Y. Bruynseraede, Czech. J. Phys. 46, 2337 (1996); V. Bruyndocx, C. Strunk, V. V. Moshchalkov, C. Van Haesendonck, and Y. Bruynseraede, Czech. J. Phys. 46, 2339 (1996).

6. C. C. Abilio, P. Butand, T. Fournier, B. Pannetier, J. Vidal, S. Jedesko, and B. Dalzotto, Phys. Rev. Lett. 83, 5102 (1999).

7. W. A. Little and R. D. Parks, Phys. Rev. Lett. 9, 9 (1962).

8. R. P Groff and R. D. Parks, Phys. Rev. 176, 567 (1968).

9. S. Alexander, Phys. Rev. B27, 1541 (1983).

10. S. Alexander and E. Halevi, J. Phys (Paris) 44, 805 (1983).

11. P. G. de Gennes, C. R. Acad Sci. 292, 9 (1981).

12. V. M. Vinokur, JETP 66, 198 (1987).

13. L. L. Shohn and M. Tinkham, Phys. Rev. B47 97; ibid. B47, 967 (1993).

14. Y. Aharonov and D. Bohm, Phys. Rev. 115, 485 (1959).

15. A. G. Aronov and Y. S. Sharvin, Rev. Mod. Phys. 59, 755 (1987).

16. I. V. Krive and A. S. Rozhavsky, Int. J. Mod. Phys. 6, 1255 (1992).

17. C. N. Yang, Rev. Mod. Phys. 34, 694 (1962).

18. E. N. Bogachek, G. A. Gogadze, and I. O. Kulik, Phys. Status Solidi (b) 67, 287 (1975).
20. V. M. Gvozdikov, Sov. Phys. Solid State 26, 1560 (1984); ibid. 28, 179 (1986).

21. V. M. Gvozdikov, Fiz. Nizk. Temp. 18, 1128 (1992).

22. J. R. Schrieffer, Theory of Superconductivity, Nauka, Moscow (1970).

23. J. Bardin, R. Kummel, A. E. Jacobs, and L. Tewordt, Phys. Rev. 187, 556 (1969).

24. I. S. Gradshtein and I. M. Ryzhik, Tables of Integrals, Sums, Series, and Products, Nauka, Moscow (1971) (in Russian).

25. V. M. Gvozdikov and M. V. Gvozdikova, Phys. Rev. B58, 8716 (1998)

26. V. M. Gvozdikov and E. Manninen, Fiz. Nizk. Temp. 6, 1138 (1980) [Sov. J. Low Temp. Phys. 6, 552 (1980)].

27. I. O. Kulik, JETP Lett. 11, 275 (1970).

28. T. J. B. Janssen, N. Harrison, S. M. Hyden, P. Meeson, and M. Springford, Phys. Rev. B57, 11698 (1998).

29. T. Maniv, V. N. Zhuravlev, I. D. Vagner, and P. Wyder, J. Chem. Solids 59, (1998).

30. J. Rollbuhler and A. A. Odintsov, LT22 Proceedings, LT22 CDROM, paper N10 616. pdf (1999).

31. P. L. Mc Euen, M. Bockrath, D. H. Cobdeh, Y. G. Yoon, and S. G. Lois, Phys. Rev. Lett. 83, 5098 (1999).

32. L. P. Gor'kov, Zh. Eksp. Teor. Fiz. 36, 1918 (1959).

33. H. F. Cheung, Y. Gefen, E. K. Reidel, and W. H. Shih, Phys. Rev. B37, 6050 (1988).

34. A. A. Zvyagin and I. V. Krive, Fiz. Nizk. Temp. 21, 687 (1995).

35. R. G. Chambers, Phys. Rev. Lett. 5, 3 (1960).

36. M. Buttiker, Y. Imry, and R. Landauer, Phys. Lett. A96, 365 (1983). 